\title{
Recombinant Human Voltage Dependent Anion Selective Channel Isoform 3 (hVDAC3) Forms Pores with a Very Small Conductance
}

\author{
Vanessa Checchetto ${ }^{a}$ Simona Reina ${ }^{b, c}$ Andrea Magrib,c Ildikò Szabo ${ }^{a}$ \\ Vito De Pinto ${ }^{b, c}$ \\ aDepartment of Biology, University of Padova, Padova, b Department of Biological, Geological and \\ Environmental Sciences, Section of Molecular Biology, University of Catania, 'National Institute for \\ Biomembranes and Biosystems, Section of Catania, Catania, Italy
}

\section{Key Words}

Voltage Dependent Anion selective Channel isoform 3 - Pore-formation • Mitochondrial outer membrane • Planar lipid bilayer

\begin{abstract}
Background/Aims: Voltage-dependent anion channels (VDAC), also known as eukaryotic porins, are located in the outer mitochondrial membrane and allow the flux of ions and small metabolites. While the pore-forming ability of recombinant VDAC1 and VDAC2 has been extensively studied during the last decades, a clear-cut ion conducting channel activity has not been assigned to the VDAC3 isoform. Methods: Electrophysiological characterization of the recombinant protein purified and refolded was obtained after incorporation into planar lipid bilayers. Results: Here we report for the first time that recombinant hVDAC3, upon expression in E.coli and purification-refolding, shows a channel activity with a very small conductance $(90$ pS in $1 \mathrm{M} \mathrm{KCl}$ ) with respect to the conductance of hVDAC1 (>3500 pS in $1 \mathrm{M} \mathrm{KCl).} \mathrm{Purified}$ hVDAC3 allowed the passage of both chloride and gluconate anions and did not distinguish between potassium, sodium and calcium used as cations. In contrast to VDAC1, the channel was active also at transmembrane voltages higher than $+/-40 \mathrm{mV}$ and displayed a relatively high open probability even at +/-80 mV. hVDAC3 was only slightly voltage-dependent, displaying a tendency to adopt lower-conductance states at positive voltages applied to the cis chamber. In accordance with the small conductance of the pore, expression of hVDAC3 in a porin-less yeast strain allowed only partial recovery of the growth under non-permissive conditions. Conclusion: The observed electrophysiological properties of hVDAC3 are surprisingly different from the other isoforms and are discussed in relation to the proposed physiological role of the protein in mammalian cells.
\end{abstract}

V. Checchetto and S. Reina equally contributed to the work.

Prof. Vito De Pinto

and Prof. Ildikò Szabo
Department of Biological, Geological and Environmental Sciences, Section of Molecular Biology,University of Catania Building 2, floor 3, CU S.Sofia, viale A. Doria, 6, 95125 Catania, (Italy) and Department of Biology, University of Padova, viale G. Colombo 3, 35121 Padova (Italy)

E-Mail vdpbiofa@unict.it and E-Mail ildi@civ.bio.unipd.it 
Checchetto et al.: Electrophysiology of Recombinant hVDAC3

\section{Introduction}

Voltage Dependent Anion-selective Channels (VDACs) are small proteins (30 kDa) whose most known function is to form pores in the outer mitochondrial membrane [1, 2]. Evolution yielded three distinct isoforms of these proteins, with the encoding genes located in different chromosomes. The three isoforms, named VDAC1, VDAC2 and VDAC3 in the chronological order of their discovery, show a slightly different tissue-dependent expression pattern (all three can be considered ubiquitous but VDAC3 is prevalently present in testis), a variable abundance (VDAC1 is the most abundant isoform [3, 4]), a difference in cysteine content (VDAC1 has only 2 Cys, VDAC2 9 Cys and VDAC3 6 Cys) and are characterized by specific interactome partners [5]. VDACs' functional role is strictly associated to the permeability of the outer mitochondrial membrane. These proteins form relatively wide, open pores, allowing the permeability of ions, metabolites and molecules up to a hydrated shell size smaller then the pore diameter. The possibility that such pores can be regulated by a gating mechanism has been suggested by VDAC being gated by voltage, a property easily detectable in in vitro experiments $[1,4]$. This observation raised a striking interest in VDACs because gating of the pores in the outer membrane was expected to regulate the whole bioenergetic function of mitochondria.

The VDAC isoforms show difference in their ability to support bioenergetic metabolism. VDAC1, the most studied isoform, has been deeply investigated from the functional point of view: it is accepted that VDAC1 is able to form pores allowing the flow of nucleotides (ADP and ATP), the main substrates/products of mitochondria and the most important result of respiration. As a matter of fact, deletion of the VDAC1 homologous in S. cerevisiae results in complete arrest of cell growth under restrictive conditions, i.e. in the presence of a pure respiratory substrate like glycerol and at a temperature of $37^{\circ} \mathrm{C}[6]$. This deletion mutant has thus been used to establish a complementation assay that provides indications about the function of isoforms or mutants of VDAC. While transformation with VDAC1 and VDAC2 from other eukaryotes (i.e. human) is able to restore the normal growth of $\Delta$ porin yeast, VDAC3 is able to do so only to very little extent [7]. This information led to the hypothesis that VDAC3 is not able to form pores. Furthermore, an early report using VDAC3 purified from mitochondria of yeast transformed with the corresponding cDNA indeed stated that no activity at all or very seldom pore insertion can be detected [8].

From the structural point of view all three isoforms are expected to share several elements. The structure of VDAC1 has been determined using different techniques (both NMR [9], X-ray crystallography [10] and a mix of these two [11]), leading to a strong, coherent model in terms of secondary structure architecture. According to Ujwal and colleagues, the $\alpha$-helix of the $\mathrm{N}$-terminal segment is oriented against the interior wall, causing a partial narrowing at the center of the pore, and thus possibly regulating the conductance of ions and metabolites passing through the VDAC pore. The topography of VDAC1 in the mitochondrial outer membrane has been determined in intact cells [12], revealing that the $\mathrm{N}$-terminus is located versus the cytosol. Recently the structure of zebrafish VDAC2 has been determined at $2.8 \AA$ resolution, confirming the general organization of the pore [13]. The predictive homology modelling of VDAC3 confirms that, similarly to VDAC1, its transmembrane domain should consist of up-and-down $\beta$-barrels, but leaves unsolved the problem of the $\mathrm{N}$-terminal domain and of the loop arrangement. Overall, structural similarities between the three isoforms suggest a pore-forming ability for all of them, although the properties of these pores might well be different.

In the present work we expressed the human VDAC3 sequence in E. coli and purified and refolded the proteins using the same protocol that has been successfully used to prepare VDAC1 for NMR or crystallization experiments. Subsequent electrophysiological characterization revealed that VDAC3 reproducibly form pores even though with a much smaller conductance than the other two isoforms. 


\section{Materials and Methods}

Yeast strains and growth conditions

Wild type Saccharomyces cerevisiae strain BY4742 [MAT a, his $3 \Delta 1$, leu $2 \Delta 0$, lys $2 \Delta 0$, ura3 $\Delta 0$ ] and isogenic porin-depleted mutant $\Delta$ por1 [MATa, his $3 \Delta 1$, leu2 $\Delta 0$, lys $2 \Delta 0$, ura3 $\Delta 0$, por1::kanMX4] were obtained from EUROSCARF (Frankfurt, Germany). Cells were grown in YP (1\% yeast extract, 2\% peptone) supplemented with $2 \%$ glucose (YPD) or in minimal medium ( $0,67 \%$ yeast nitrogen base without amino acids) containing $2 \%$ glucose (SD) supplemented with $20 \mu \mathrm{g} / \mathrm{mL}$ of the appropriate nutritional requirements according to the genotype of the strains at $28^{\circ} \mathrm{C}$. Solid media were supplemented with $2 \%$ Bactoagar (Difco).

Human VDAC proteins (hVDAC1 and hVDAC3) were cloned in pYX212 shuttle vector and introduced into yeast $\Delta$ por1 cells by lithium-acetate method, as already reported in $[3,14]$. Transformants were selected on media containing essential nutrients except uracil and used for drop serial dilution assays.

Cloning, heterologous expression and purification of hVDACs

hVDAC1 (used as an internal control) and hVDAC3 genes were cloned into the pET21a vector (Novagen) using NdeI/XhoI sites. E. coli BL21(DE3) cells were transformed with pET21a harboring the hVDAC genes. Transformants were grown in LB medium containing ampicillin $\left(100 \mu \mathrm{g} \cdot \mathrm{ml}^{-1}\right)$. Induction was performed by addition of $1 \mathrm{mM}$ isopropyl- $\beta$-D-thiogalactopyranoside (IPTG) at an optical density ( $\lambda=595 \mathrm{~nm}$ ) of $\sim 0.6$ as previously described in [14]. Proteins were purified in urea by chromatography using nickel-nitrilotriacetic acid resin (Ni-NTA, Qiagen) [14].

\section{Protein Refolding}

The denatured protein mixture was added drop-wise to $25 \mathrm{mM}$ TrisHCl (pH 7.0), $100 \mathrm{mM} \mathrm{NaCl}, 1 \mathrm{mM}$ EDTA, $1 \%(\mathrm{v} / \mathrm{v})$ lauryldimethylamine-oxide (LDAO), allowing a ten-fold dilution of the urea concentration, and the mixture was gently stirred overnight at $4^{\circ} \mathrm{C}$. The protein solution was then dialyzed against a $100 \times$ buffer containing $0.1 \%$ LDAO overnight at $4{ }^{\circ} \mathrm{C}$. Thermo Scientific Slide-A-Lyzer Dialysis Cassettes (3.5 K MWCO) were used to avoid any sample loss. The protein purity was verified by 12\% SDS-PAGE and Comassie staining. Purified samples were stored at $4^{\circ} \mathrm{C}$ until further use.

\section{VDAC channel reconstitution, recording and analysis}

Refolded VDAC proteins were reconstituted into a PLB (planar lipid bilayer). Single-channel current recording and data analysis were carried out as described previously [15]. Briefly, a Warner Instruments (Hamden) electrophysiological planar bilayer apparatus was used and bilayers were prepared using asolectin dissolved in decane (Sigma) containing 1\% chloroform (Sigma) across a $250 \mu \mathrm{M}$ hole in a polystyrene cuvette (Warner Instruments). Asolectin was partially purified by precipitation with cold acetone from a chloroform solution. The lipid membrane divided the trans and the cis compartments. When a bilayer membrane was formed, currents across the membrane elicited by different voltage pulses were measured and membrane resistance could be determined. A bilayer membrane was considered to be satisfactory for further experiment if it exhibited an approximately 150-200 pF capacity. The volumes of the cis and trans compartments were $3 \mathrm{ml}$. Solution in the chambers could be mixed through stirring with a small magnetic bar. Both sides were connected to the electrodes via salt bridges $(1 \mathrm{M} \mathrm{KCl})$ in series with $\mathrm{Ag} / \mathrm{AgCl}$ electrodes. All measurements were made at room temperature.

The standard experimental medium was $1 \mathrm{M} \mathrm{KCl}, 10 \mathrm{mM}$ Hepes/pH 7.2. Otherwise, $1 \mathrm{M}$ Kgluconate, 1 $\mathrm{M}$ Na gluconate or $500 \mathrm{mM}$ Calcium chloride were used. Control experiments with empty membrane or with detergents used for the purification showed no activity in any of the above solutions. All voltages reported are those of the cis chamber, zero being assigned by convention to the trans (grounded) side. Currents are considered as positive when carried by cations flowing from the cis to the trans compartment. Data were acquired using a Bilayer Clamp amplifier (Warner Instruments, USA) at $100 \mu \mathrm{s} /$ point, filtered at $300 \mathrm{~Hz}$ and analyzed offline using the pClamp program set (Axon Instruments, Union City, CA, USA). Histograms were fitted using Origin7.5 program set. Leak was not substracted. 
Fig. 1. hVDAC3 only partially complements a yeast strain lacking porin. A) Serial dilution of yeast colony, showing their relative growth, in different conditions. The carbon source was, respectively, glucose or glycerol and the incubation temperature $28^{\circ} \mathrm{C}$ or $37^{\circ} \mathrm{C}$. As a control, BY4742, a wild type strain, $\Delta$ por 1 , a strain devoid of endogenous porin $1[3,28]$ growth in the absence or after transformation with hVDAC1 or hVDAC3. B) Serial dilution of yeast colony, showing their relative growth, in the presence of $60 \mathrm{mM}$ acetic acid.

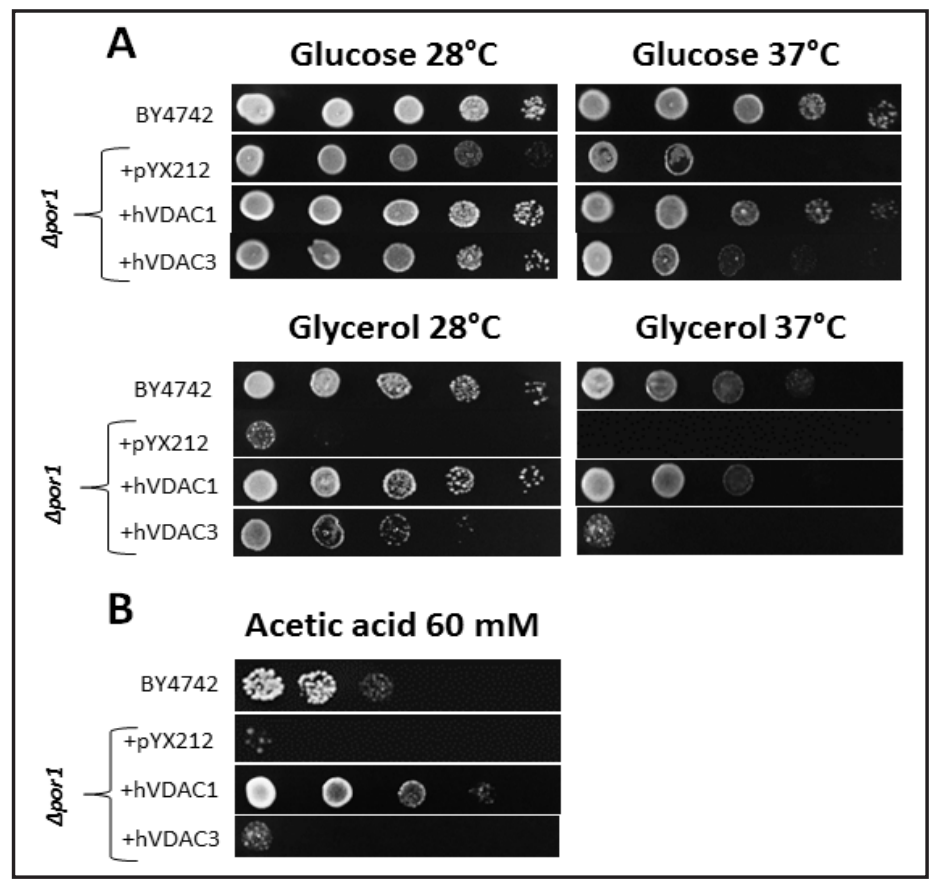

\section{Results}

Cellular impact of the exchange of yeast porin1 with hVDAC3

We compared the function of human VDAC1 and VDAC3 isoforms expressed in yeast, to assess whether the two proteins have a different behaviour when inserted into yeast mitochondria devoid of the endogenous porin1. Human VDAC3 (hVDAC3) and hVDAC1, cloned in a yeast shuttle vector, were thus used to complement the growth defect associated with the inactivation of the $S$. cerevisiae endogenous porin $1[6,7]$. In the panels of Figure $1 \mathrm{~A}$, it is shown that while human VDAC1 is able to fully complement the growth defect, hVDAC3 is not able to give a comparable phenotype in non permissive conditions, like a temperature of $37^{\circ} \mathrm{C}$ and non fermentable glycerol, as a sole carbon source. Under these conditions glycerol can serve only as respiratory substrate and it cannot be exploited for glycolysis. Only very little growth can be detected in the most concentrated sample (Fig. 1A). However, also in glucose as a carbon source, at $37^{\circ} \mathrm{C}$ the transformation with hVDAC3 allows recovery to a smaller extent than with hVDAC1. This finding is consistent on one hand with glucose being used in part for glycolysis and thus producing small amount of ATP in the cytosol and on the other hand with a reduced metabolite flux across the OMM. Addition of $60 \mathrm{mM}$ acetic acid (Fig. 1B), an apoptotic inducer for yeast, in the culture medium replicates the difference between the complementation with hVDAC1 and hVDAC3. In general while hVDAC1 is able to replicate the behaviour of the wild type $S$. cerevisiae, hVDAC3 gives only a very limited support to the $\Delta$ porin1 yeast strain.

\section{Electrophysiological characterization of recombinant purified hVDAC3}

In order to test the activity of hVDAC3 by a more direct approach, we cloned the sequences of the two human VDACs (hVDAC1 and hVDAC3) in an E. coli specific expression vector. The proteins, containing a C-terminal $6 x$ His tag, were obtained from E. coli inclusion bodies, denatured in urea and then isolated by chromatography using a Ni-NTA column (Fig. 2A-B). Next, they were refolded using a dialysis protocol, as described in the Materials and Methods section. As shown in Figure 2B, this protocol yielded a substantial amount of the expressed protein upon induction, and the eluted protein fractions did not contain significant contaminations. Both VDAC1 and VDAC3 migrated with a molecular weight (32.5 and 30.5 $\mathrm{kDa}$, respectively) close to their predicted MWs (31.84 and $31.73 \mathrm{kDa}$ respectively). 
Fig. 2. Expression of recombinant human VDACs. A) Expression of hVDAC1 and hVDAC3. M: Marker; 1 : non-induced lysate; 2: hVDAC1 expression; 3: noninduced lysate; 4: hVDAC3 expression. B) Purification of hVDAC1 and hVDAC3 by Ni-NTA chromatography. M: Marker; 1: purified recombinant hVDAC1; 2:purified recombinant hVDAC3. The two proteins show a different electrophoretic mobility despite the almost identical molecular weight.
Fig. 3. Recombinant human VDAC1 form channels with typical biophysical characteristics. Upper part: VDAC1 activity recorded in $1 \mathrm{M} \mathrm{KCl}$ medium upon application of the indicated voltage pulses. Current steps corresponding to $3.5 \mathrm{nS}$ conductance are observable. Lower part: activity of human recombinant VDAC3 obtained with the same purification protocol. The same scale was used for comparison. Current traces are representative of 4 (upper part) and 35 (lower part) similar experiments.
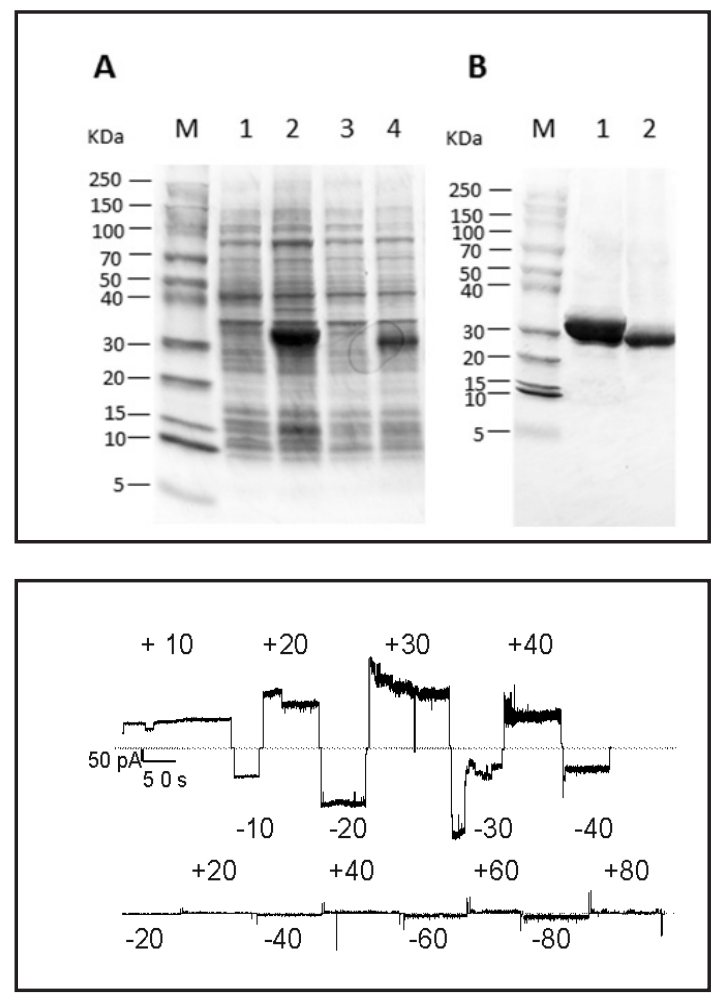

Fig. 4. VDAC3 forms small channels. Left panel: Current traces recorded in $1 \mathrm{M} \mathrm{KCl}$ medium at + and - 30 $\mathrm{mV}$ applied voltages (cis side) using purified VDAC1 (representative of 4 experiments). The tendency to close at both potentials is clearly observable. Lower trace: activity at $-30 \mathrm{mV}$ on extended time and current scale. Right panel: VDAC3 activity recorded under the same conditions as VDAC1 (left panel). Please note that the current flowing through VDAC3 is much smaller than through VDAC1 at both potentials and VDAC3 does not show

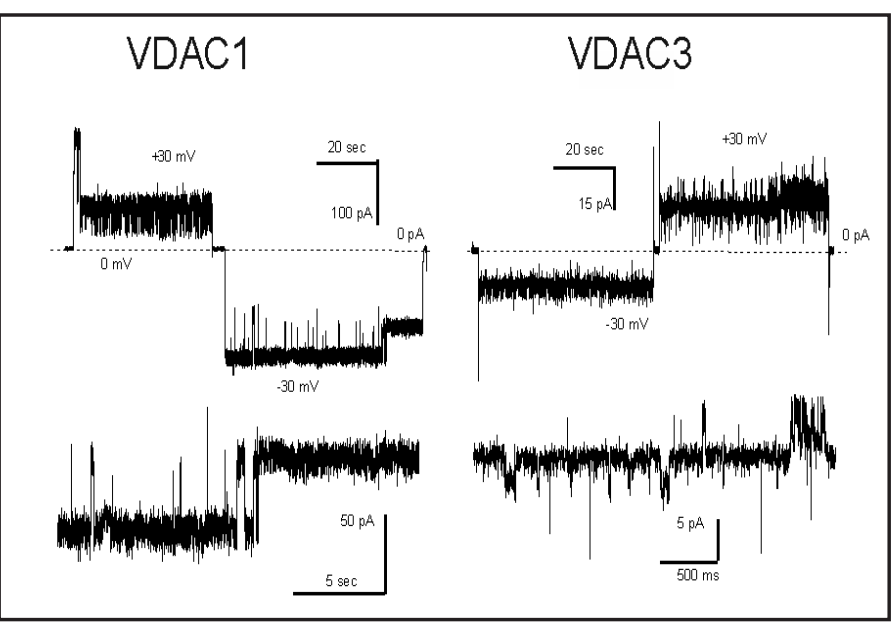
a tendency to adopt partially open states. Lower trace: activity at $+30 \mathrm{mV}$ on extended time and current scale.

The purified and refolded proteins obtained from two different preparations were then used for electrophysiological characterization. First, we confirmed that hVDAC1 obtained with the above described protocol gave channel activity showing main characteristics that are compatible with those previously described for this protein $[16,17]$.

As shown in the representative experiment (Fig. 3A), hVDAC1 gave rise to a channel activity with $3.5 \mathrm{nS}$ conductance in $1 \mathrm{M} \mathrm{KCl}$, as expected. Furthermore, hVDAC1 showed the typical voltage-dependent behavior, i.e. it adopted partially closed states already at +/$30 \mathrm{mV}$. Observation of these typical characteristics of hVDAC1 confirmed the validity of our purification protocol. Therefore, we proceeded with the study of hVDAC3, which was obtained in the same way. Under the same ionic conditions, hVDAC3 displayed a very small 


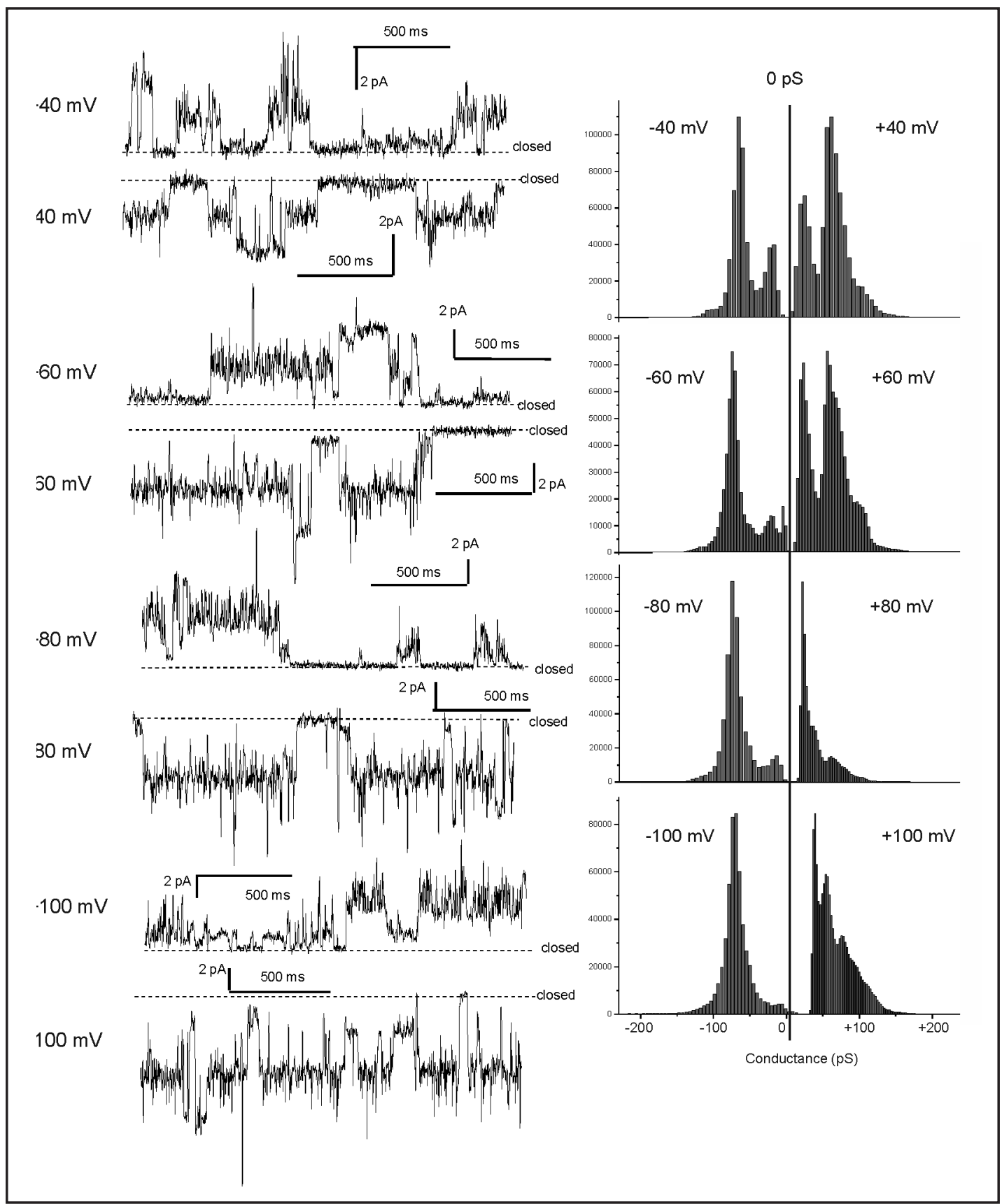

Fig. 5. VDAC3 is active at high membrane potentials. Left panels: Representative current traces from the same experiment recorded in $1 \mathrm{M} \mathrm{KCl}$ at the indicated membrane potentials (cis side). Right panels: Corresponding amplitude histograms obtained from $60 \mathrm{sec}$ recordings using the PCLAMP 8.0 analysis program. Y scale shows the number of events recorded. X scale shows the conductance, obtained by dividing the measured current levels with the applied voltages. Please note that the conductance remained constant at negative potentials in the range of -40 to $-100 \mathrm{mV}$, while at positive potentials lower conductance states were adopted at voltages higher than $+60 \mathrm{mV}$. This result is representative of 17 independent experiments recorded in various ionic conditions.

activity when compared to hVDAC1 (Fig. 3B) and the activity persisted also at high membrane potentials. The lack of tendency of adopting a closed state upon application of $+/-30 \mathrm{mV}$ is even more evident in Figure 4, which shows representative current traces recorded from other two experiments using hVDAC1 and hVDAC3. Indeed, analysis of another representative 
Fig. 6. VDAC3 shows conductance substates. Activity recorded in $1 \mathrm{M} \mathrm{KCl}$ at $+100 \mathrm{mV}$. Full-conductance openings and closures are observable. In addition, closures to different subconductance states are evident (arrows). Similar activity was observed in basically all experiments performed with VDAC3 (n=35 in $\mathrm{KCl}$ ) (see also e.g. current traces in Fig 5.).

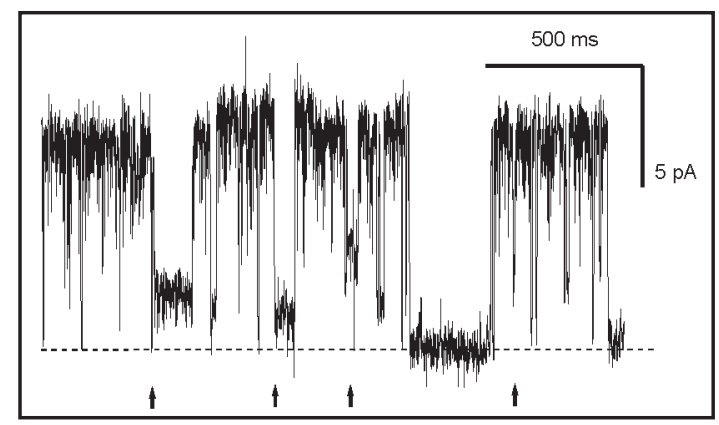

Fig. 7. VDAC3 does not discriminate between $\mathrm{KCl}$ and Kglcuconate. Upper part: Representative current traces recorded at $-100 \mathrm{mV}$ (1 M $\mathrm{KCl})$ and at $-80 \mathrm{mV}(1 \mathrm{M}$ Kgluconate $)(\mathrm{n}=4)$. Lower part: Current-voltage relationships in $1 \mathrm{M} \mathrm{KCl}$ and $1 \mathrm{M}$ Kgluconate. At each voltage, current levels were measured from 3 different experiments performed under the same condition. Mean \pm SD are shown $(n=30$ to 50 for each point). Fitting was obtained using the Origin 6.1 Program set and gave slope conductances of 90 and $108 \mathrm{pS}$ in $1 \mathrm{M} \mathrm{KCl}$ and $1 \mathrm{M}$ Kgluconate, respectively.

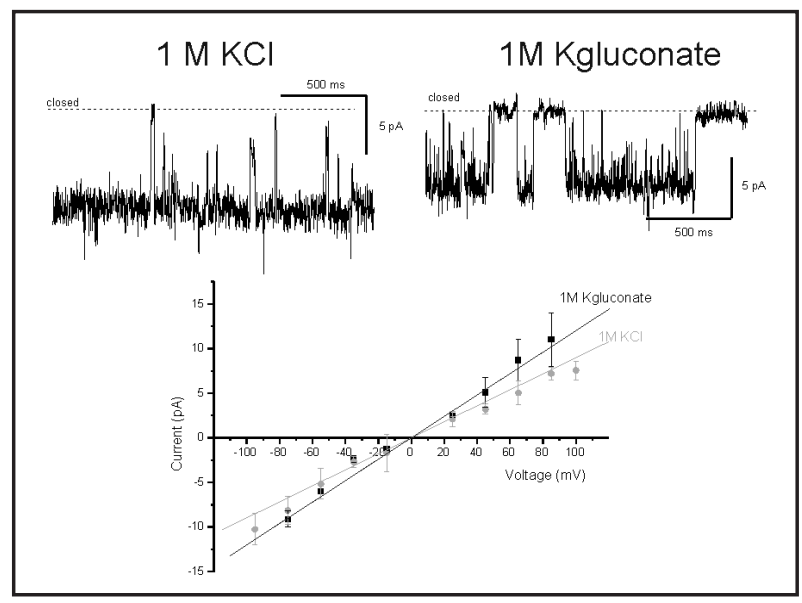

Fig. 8. VDAC3 activity in different ionic conditions. Current traces recorded at + or $-80 \mathrm{mV}$ voltage pulses in $1 \mathrm{M} \mathrm{KCl}, 1 \mathrm{M}$ Kgluconate $(\mathrm{n}=4)$ and $1 \mathrm{M} \mathrm{Na}$ gluconate $(n=4)$ are shown. Pulses from $-80 \mathrm{mV}$ to 0 and then from 0 to $+80 \mathrm{mV}$ are shown. In all three mediums VDAC3 displayed similar conductances, fast flickering behavior and tendency to adopt closed state especially at positive potentials.

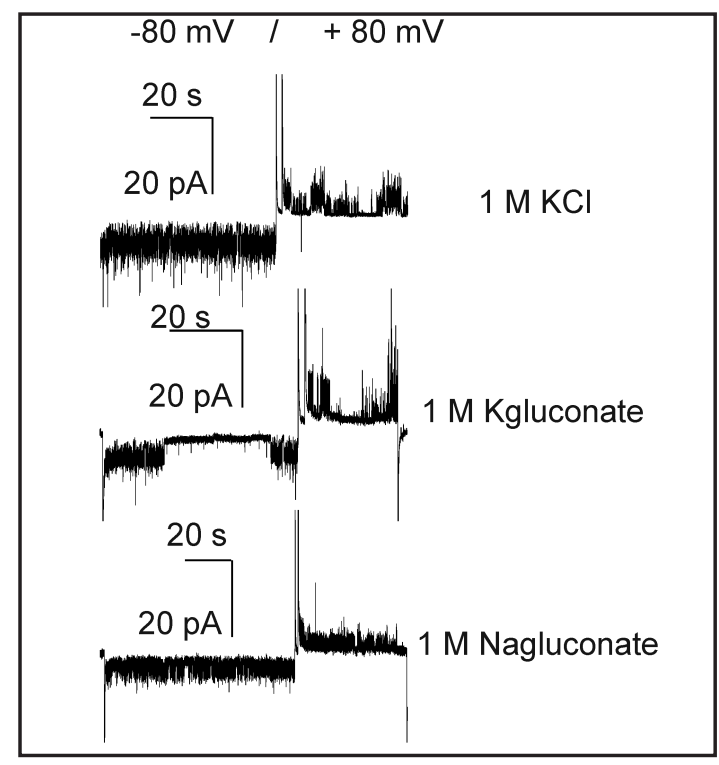

experiment reveal that hVDAC3 is active also at very high potentials, ranging up to $100 \mathrm{mV}$ (Fig. 5). The respective amplitude histograms indicate that hVDAC3 showed a tendency to adopt lower-conductance states prevalently at positive voltages. The peculiar property of hVDAC3 shown in Figure 5 was observed in all experiments where higher voltages were applied $(\mathrm{n}=17)$. Figure 6 shows occurrence of numerous conductance substates, as typical also of hVDAC1 and interpreted as a consequence of the $\beta$-barrel structure of these pores.

Next, we studied the conductance and the voltage-dependence of hVDAC3 recorded in different ionic conditions. Figure 6 illustrates activities observed in $1 \mathrm{M} \mathrm{KCl}$ or in $1 \mathrm{M}$ Kgluconate. Analysis of the current-voltage relationship did not reveal a statistically significant difference in conductance. The slope conductances were $90 \mathrm{pS}(1 \mathrm{M} \mathrm{KCl})$ and 108 
Fig. 9. VDAC3 activity in $500 \mathrm{mM}$ Calcium chloride. Activity is shown at the indicated potentials. In this medium, containing $1 \mathrm{M}$ chloride anions, the conductance was approx. $100 \mathrm{pS}$, as in the case of $1 \mathrm{M}$ $\mathrm{KCl}$. Current traces are represenatative of 4 similar experiments.

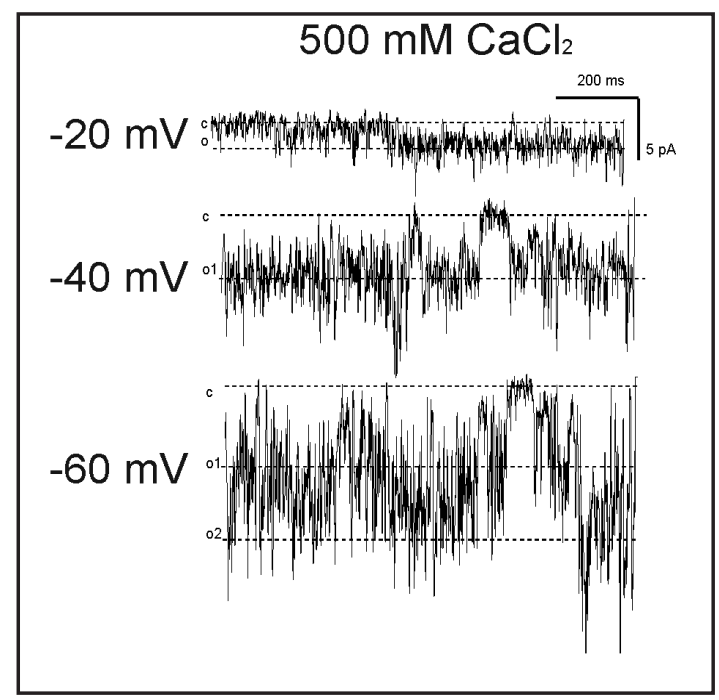

Fig. 10. Open probability of VDAC3 in $1 \mathrm{M}$ Kgluconate at various potentials. Open probability as a function of time was monitored and measured for the indicated times using the PCLAMP 8.0 program set. A decrease of the open probability can be observed at higher potentials.
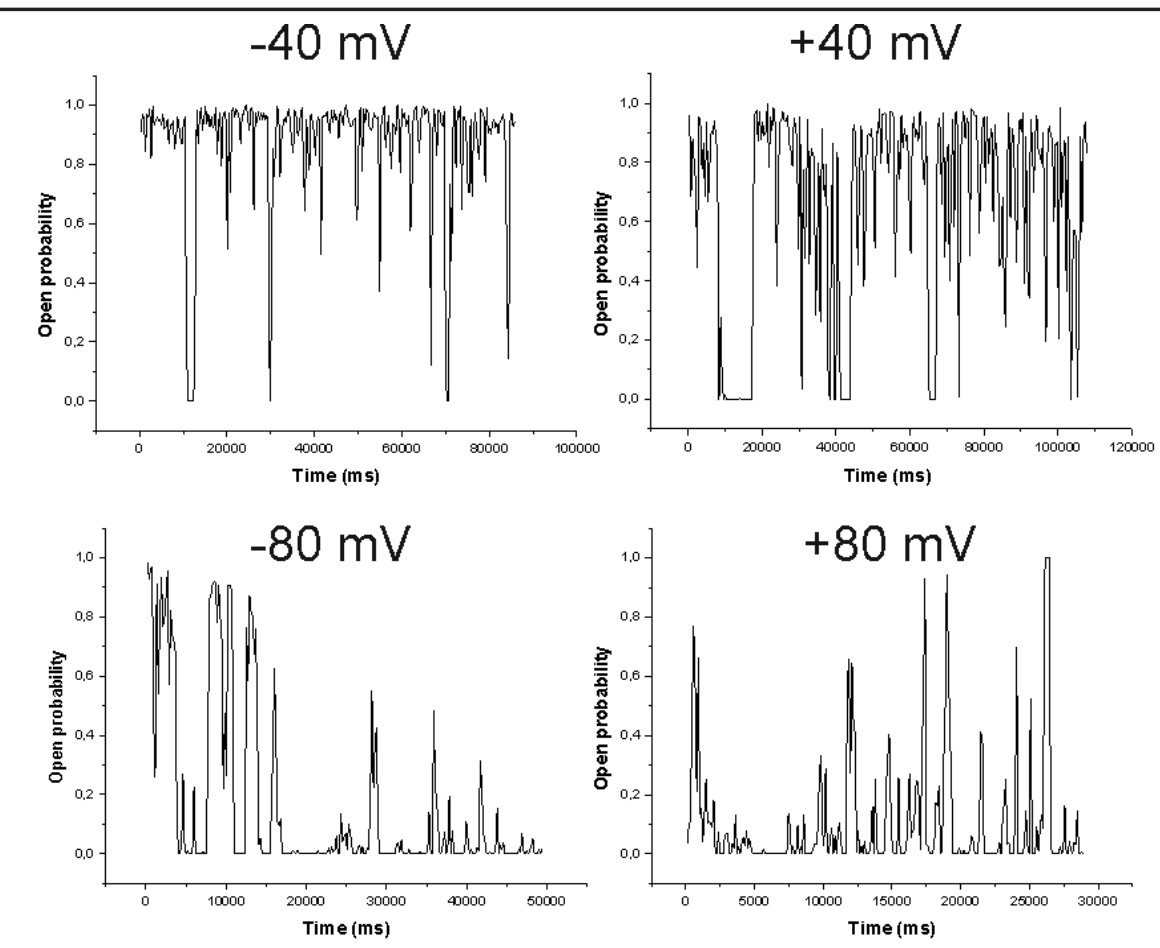

pS (in $1 \mathrm{M}$ Kgluconate) as revealed by fitting the I-V curves obtained from three different experiments for each condition. Furthermore, conductance and voltage-dependence were comparable both in $1 \mathrm{M}$ Kgluconate and in $1 \mathrm{M}$ Na gluconate (Fig. 8). Calcium has been shown to regulate hVDAC1 activity and permeability [18] and to selectively transfer calcium signal to mitochondria during apoptotic signal [19]. In our case, hVDAC3 channel activity was observable with the predicted conductance also in calcium chloride, although a fast flickering behavior became predominant (Fig. 9). Finally, the open probabilities obtained in $1 \mathrm{M}$ Kgluconate at various potentials are shown in Figure 10, indicating that the open probability decreased by increasing the membrane potential but the channel was not closed for long periods even at $+/-80 \mathrm{mV}$. Thus, the ability of hVDAC3 to adopt open states at high negative and positive potentials is conserved, independently of the conducting ions used in this study. 
Checchetto et al.: Electrophysiology of Recombinant hVDAC3

\section{Discussion}

The sequence of hVDAC3 is highly homologous to those of the isoforms hVDAC1 and 2 , and bioinformatic predictions [3] have indicated that secondary and tertiary structures of hVDAC3 are similar to that of hVDAC1 $[9,10,11]$. The homology is also reflected in the close evolutionary relationships with the other two isoforms: VDAC1 and VDAC2 seems to have evolved from VDAC3, considered to be the oldest isoform [4, 20]. Furthermore, genetic analysis has shown that even though located in different chromosomes, the VDAC3 gene is similar to VDAC1 and VDAC2 genes. Indeed they share exactly the same exon-intron organization and a strictly conserved splicing-sites pattern [21]. The overall information briefly summarized here prompted the scientific community to consider a priori VDAC3 as an additional pore-forming protein.

Despite this apparently straightforward picture, VDAC3 can be considered the most elusive isoform in the family of VDAC proteins. The first surprising result was obtained when functional assays were used to track its specificity. The complementation assay in the $S$. cerevisiae $\Delta$ porin 1 strain soon revealed that VDAC3 is not able to fully recover the growth features of the yeast, at variance with VDAC1 and 2 [7]. Indeed, VDAC3 allows a very scarce growth in conditions of strict oxidative metabolism: this means, in terms of complementation, that VDAC3 is targeted to the yeast mitochondria but it is able to restore the bioenergetic metabolism only partially or very poorly. Being at the interface between mitochondria and the cytosol, VDAC regulation potentially defines the fate of mitochondria: respiration and viability or apoptosis and cell death [1]. Previous findings support the hypothesis that adenine nucleotides flow through VDAC and that VDAC gating might regulate the permeability of the outer membrane and thus control mitochondrial function. Indeed, Colombini and co-workers found that a change in the pore diameter from $2.5-3 \mathrm{~nm}$ in the open state to $1.7-1.8 \mathrm{~nm}$ in the closed state of VDAC1 resulted in an almost completely abolished ATP flux through the isolated and reconstituted channel [22]. The authors also concluded that the pore diameter reduction cannot by itself account for changes in ATP permeability, rather, conformational changes would cause a change in the net charge in the pore wall from positive to negative upon partial closure. Thus, exclusion of ATP would be the result of an electrostatic barrier [22]. Hiller proposed that the movement of N-terminal sequence, a domain standing inside the pore and partially occluding the hollow cylindrical barrel, could operate as a gate: by this motion the N-terminal domain would restrict the pore lumen to the nucleotides [23].

Previous attempts to characterize the activity of VDAC3 were unsuccessful. Expression of the protein in S. cerevisiae and its purification starting from the yeast mitochondria did not result in an activity comparable to those of VDAC1 and VDAC2, i.e. a large pore conductance and a typical voltage dependence [8]. Here we report for the first time that VDAC3 is able to form small-conductance channels in $1 \mathrm{M} \mathrm{KCl}$ in a reproducible way. VDAC3 activity might have been previously overlooked because of this small conductance. In a fraction enriched in VDAC2 and 3, obtained from bovine spermatozoa, an electrophysiological activity coherent with that of VDAC2 alone has been observed [24]. Recently, Maldonado et al. isolated mitochondrial fractions of VDAC2/3, VDAC1/3, and VDAC1/2 double silenced HepG2 cells to yield VDAC1, VDAC2, and VDAC3-enriched fractions, respectively [25]. Since silencing does not produce a complete knockdown of the targeted genes, contribution of VDAC1 and VDAC2 isoforms to the observed activity cannot be excluded.

According to Laplace law $\left(\mathrm{G}=\sigma \pi \mathrm{r}^{2} / I\right)$, where $\mathrm{G}$ is the measured conductance of the channel, $\sigma$ is the specific conductance of the aqueous phase $(112 \mathrm{mS} / \mathrm{cm}$ for $1 \mathrm{M} \mathrm{KCl})$ and $l$ is the length of the channel (generally considered $7.5 \mathrm{~nm}$ for the $\mathrm{OMM}$ ), it is possible to estimate [26] the diameter of the VDAC3 pore to be approx. $0.3 \mathrm{~nm}$. The same calculation for VDAC1 indicated a diameter of 2.5-3 nm [27]. Even though these estimations are not correct, as we know now from the presently available structures, they are indicative of a difference in the range of one order of magnitude between the pores formed by the two proteins. The small pore diameter might well account for reduced metabolite flux across VDAC3. The question arises 
why VDAC3, having a predicted structural similarity to VDAC1 might have such a reduced conductance. The structural basis and the physiological significance of the maintenance of the open state at high voltages are also unclear. Concerning the 3-D arrangement of VDAC3, in the absence of an experimental structure, it is possible to hypothesize that some domains of the protein can play a different role in VDAC3 with respect to VDAC1. In particular we have previously demonstrated that the swapping of the N-terminal domain of VDAC3 with the same domain from VDAC1 fully restores not only the ability to complement the growth defect in $\Delta$ porin1 yeast but also confers electrophysiological properties largely overlapping those of VDAC1 to the chimera [28]. Either N1-VDAC3 purified from yeast transformed with a shuttle vector carrying the sequence of the chimera, or recombinant N1-VDAC3, extracted from E. coli and purified with Ni-NTA chromatography, are able to form large pore similar to those of VDAC1. Therefore, one possibility is that the structure/position of the N-terminal region of VDAC1 and VDAC3 are different, accounting for a partial occlusion of the pore.

As to the physiological function of VDAC3, one possibility is that this protein is not used routinely in the cell as a conduit for molecules, but it is specialized in other functions as well. The VDAC3 knock-out mouse show a peculiar phenotype: they are alive but suffer of male infertility. Such phenotype has been associated with a deficiency in the organization of the microtubules in the flagellum, possibly provoking reduced motility of the sperm [29]. Expression of VDAC3 in germinative tissues has been found in several laboratories and it has been shown to be associated to the mitochondrion, but also to other cytoskeletal structures like, for example the Outer Dense Fiber (ODF) [24]. Also in D. melanogaster there are reports showing the implication of VDACs in sperm maturation [30]. Furthermore, in the heart, the absence of VDAC3 caused a decrease in the apparent affinity of in situ mitochondria for ADP and a bioenergetics defect restricted to the heart and only to complex IV [31]. The functional alterations correlated with structural aberrations of mitochondria. These in vivo results demonstrated that, unlike VDAC1, there is muscle-type specificity for VDAC3 function and therefore in vivo these two isoforms may fulfill different physiologic functions. Accordingly, a possible involvement of VDAC3 expression and function in the early pathogenic events of the mdx muscular dystrophy in a mouse model has been proposed [32]. Altogether, the observed properties of VDAC3 might predict a compromised metabolite flux, possibly accounting for the observed phenotypes. In another recent work, convincing experiments showed the association of VDAC3 with the centriole, with a presumed "organising" role [33]. Experiments of deletion or addition of VDAC3 to the cell showed that an appropriate ciliogenesis requires VDAC3. The coordination of VDAC3 with the centrosomal protein kinase Mps1, required for the spindle assembly checkpoint, promotes ciliary disassembly during cell cycle entry and suppresses cilia assembly in proliferating cells [33]. In a mass spectrometry study aiming to identify proteins interacting with VDAC3 in live HeLa cells, components of organized subcompartments like endoplasmic reticulum (ER) and the cytoskeleton were found to interact with VDAC3 in addition to soluble cytosolic proteins [5]. ER is one of the most represented compartments. Marker proteins of the mitochondriaassociate membranes (MAM), regions required for many key cellular events, have also been identified as interacting proteins [5]. Among cytoskeletal proteins there were tubulins: it is known that tubulin is a modulator of VDAC1 and VDAC2 channel activity [5], but it has been reported that VDAC3 is less sensitive than the other isoforms to interaction with tubulin [25].

VDACs have also a pathophysiological relevance. VDACs have been involved in various cellular pathways, like the apoptotic cascade (VDAC1 isoform is considered pro-apoptotic, while VDAC2 anti-apoptotic; VDAC3 function has not yet been fully investigated in this respect $[1,4])$. In recent reports the involvement of VDACs in the control of ROS diffusion has been suggested $[28,34]$. Indeed VDACs are the exit pathways of superoxide anions from their production site at the inner mitochondrial membrane $[35,36]$. A direct connection of VDACs to some pathologies such as Parkinson's [37, 38] and Alzheimer's [39] diseases has been proposed based on high-throughput proteomic studies. 
In conclusion, our work presents an initial biophysical characterization of hVDAC3, previously suggested not to give rise to ion channel activity. Here we show that hVDAC3 reproducibly forms channels, but with a much smaller conductance than hVDAC1. Future structural and biophysical work is required to understand in detail the specific features of hVDAC3 accounting for the small conductance and the phenotype linked to its lack in yeast as well as in mouse. A study of the effect of the recently identified interaction partners [5] on hVDAC3 activity might also be useful in this respect.

\section{Acknowledgements}

The authors are grateful to the Italian Ministry (PRIN 2010CSJX4F to V.D.P. and I.S.) and to AIRC (grant 11814 to I.S) for financial support. We also thank dr. Angela Messina for useful discussion.

\section{References}

1 Shoshan-Barmatz V, De Pinto V, Zweckstetter M, Raviv Z, Keinan N, Arbel N: VDAC, a multi-functional mitochondrial protein regulating cell life and death. Mol Aspects Med 2010;31:227-285.

2 Szabo I, Zoratti M. Mitochondrial channels: ion fluxes and more. Physiol Rev 2014;94:519-608.

-3 De Pinto V, Guarino F, Guarnera A, Messina A, Reina S, Tomasello FM, Palermo V, Mazzoni C: Characterization of human VDAC isoforms: a peculiar function for VDAC3? Biochim Biophys Acta 2010;1797:1268-1275.

- Messina A, Reina S, Guarino F, De Pinto V: VDAC isoforms in mammals. Biochim Biophys Acta 2012;1818:1466-1476.

-5 Messina A, Reina S, Guarino F, Magrì A, Tomasello F, Clark RE, Ramsay RR, De Pinto V: Live cell interactome of the human voltage dependent anion channel 3 (VDAC3) revealed in HeLa cells by affinity purification tag technique. Mol Biosyst 2014. [Epub ahead of print].

6 Blachly-Dyson E, Zambronicz A, Hong Yu W, Adams V, McCabe ERB, Adelman J, Colombini M, and Forte M: Cloning and functional expression in yeast of two human isoforms of the outer mitochondrial membrane channel, the Voltage-dependent Anion Channel. J Biol Chem 1993;268:1835-1841.

7 Sampson MJ, Lovell RS, Craigen WJ: The murine voltage-dependent anion channel gene family. Conserved structure and function. J Biol Chem 1997;272:18966-18973.

-8 Xu X, Decker W, Sampson MJ, Craigen WJ, Colombini M: Mouse VDAC isoforms expressed in yeast: channel properties and their roles in mitochondrial outer membrane permeability. J Membr Biol 1999;170:89-102.

-9 Hiller S, Garces RG, Malia TJ, Orekhov VY, Colombini M, Wagner G: Solution structure of the integral human membrane protein VDAC-1 in detergent micelles. Science 2008;321:1206-1210.

10 Ujwal R, Cascio D, Colletier JP, Faham S, Zhang J, Toro L, Ping P, Abramson J: The crystal structure of mouse VDAC1 at 2.3 A resolution reveals mechanistic insights into metabolite gating. Proc Natl Acad Sci U S A 2008;105:17742-17747.

11 Bayrhuber M, Meins T, Habeck M, Becker S, Giller K, Villinger S, Vonrhein C, Griesinger C, Zweckstetter M, Zeth K: Structure of the human voltage-dependent anion channel. Proc Natl Acad Sci U S A 2008;105:15370-15375.

12 Tomasello MF, Guarino F, Reina S, Messina A, De Pinto V: The voltage-dependent anion selective channel 1 (VDAC1) topography in the mitochondrial outer membrane as detected in intact cell. PLoS One 2013;8:e81522.

-13 Schredelseker J, Paz A, López CJ, Altenbach C, Leung CS, Drexler MK, Chen JN, Hubbell WL, Abramson J. High resolution structure and double electron-electron resonance of the zebrafish voltage-dependent anion channel 2 reveal an oligomeric population. J Biol Chem 2014;289:12566-77.

- 14 Reina S, Magrì A, Lolicato M, Guarino F, Impellizzeri A, Maier E, Benz R, Ceccarelli M, De Pinto V, Messina A: Deletion of $\beta$-strands 9 and 10 converts VDAC1 voltage-dependence in an asymmetrical process. Biochim Biophys Acta 2013;1827:793-805.

-15 Carraretto L, Formentin E, Teardo E, Checchetto V, Tomizioli M, Morosinotto T, Giacometti GM, Finazzi G, Szabó I: A thylakoid-located two-pore K+ channel controls photosynthetic light utilization in plants. Science 2013;342:114-118.

-16 Colombini M, Blachly-Dyson E, Forte M: VDAC, a channel in the outer mitochondrial membrane. Ion Channels 1996;4:169-202. 
Checchetto et al.: Electrophysiology of Recombinant hVDAC3

17 Yu WH, Forte M: Is there VDAC in cell compartments other than the mitochondria? J Bioenerg Biomembr 1996;28:93-100.

-18 Báthori G, Csordás G, Garcia-Perez C, Davies E, Hajnóczky G. Ca2+-dependent control of the permeability properties of the mitochondrial outer membrane and voltage-dependent anion-selective channel (VDAC). J Biol Chem 2006;281:17347-17358.

19 De Stefani D, Bononi A, Romagnoli A, Messina A, De Pinto V, Pinton P, Rizzuto R: VDAC1 selectively transfers apoptotic Ca2+ signals to mitochondria. Cell Death Differ 2012;19:267-273.

20 Young MJ, Bay DC, Hausner G, Court DA: The evolutionary history of mitochondrial porins. BMC Evol Biol 2007;7:31.

21 Messina A, Guarino F, Oliva M, van den Heuvel LP, Smeitink J, De Pinto V: Characterization of the human porin isoform 1 (HVDAC1) gene by amplification on the whole human genome: A tool for porin deficiency analysis. Biochem Biophys Res Commun 2000;270:787-792.

-22 Rostovtseva T, Colombini M: ATP flux is controlled by a voltage-gated channel from the mitochondrial outer membrane. J Biol Chem 1996;271:28006-28008.

23 Hiller S, Wagner G: The role of solution NMR in the structure determinations of VDAC-1 and other membrane proteins. Curr Opin Struct Biol 2009;19:396-401.

24 Hinsch KD, De Pinto V, Aires VA, Schneider X, Messina A, Hinsch E: Voltage-dependent anion-selective channels VDAC2 and VDAC3 are abundant proteins in bovine outer dense fibers, a cytoskeletal component of the sperm flagellum. J Biol Chem 2004;279:15281-15288.

25 Maldonado EN, Sheldon KL, DeHart DN, Patnaik J, Manevich Y, Townsend DM, Bezrukov SM, Rostovtseva TK, Lemasters JJ: Voltage-dependent anion channels modulate mitochondrial metabolism in cancer cells: regulation by free tubulin and erastin. J Biol Chem 2013;288:11920-11929.

26 Benz R, Ishii J, Nakae T: Determination of ion permeability through the channels made of porins from the outer membrane of Salmonella typhimurium in lipid bilayer membranes. J Membr Biol. 1980; 56:19-29.

27 De Pinto V, Tommasino M, Benz R, Palmieri F: The 35 kDa DCCD-binding protein from pig heart mitochondria is the mitochondrial porin. Biochim Biophys Acta 1985;813:230-242.

28 Reina S, Palermo V, Guarnera A, Guarino F, Messina A, Mazzoni C, De Pinto V: Swapping of the N-terminus of VDAC1 with VDAC3 restores full activity of the channel and confers anti-aging features to the cell. FEBS Lett 2010;584:2837-2844.

-29 Sampson MJ, Decker WK, Beaudet AL, Ruitenbeek W, Armstrong D, Hicks MJ, Craigen WJ: Immotile sperm and infertility in mice lacking mitochondrial voltage-dependent anion channel type 3. J Biol Chem 2001;276:39206-39212.

-30 Guarino F, Specchia V, Zapparoli G, Messina A, Aiello R, Bozzetti MP and De Pinto V: Expression and localization in spermatozoa of the mitochondrial porin isoform 2 in Drosophila melanogaster. Biochem Biophys Res Commun 2006;346:665-670.

-31 Anflous-Pharayra K, Lee N, Armstrong DL, Craigen WJ: VDAC3 has differing mitochondrial functions in two types of striated muscles. Biochim Biophys Acta 201;1807:150-156.

- 32 Massa R, Marliera LN, Martorana A, Cicconi S, Pierucci D, Giacomini P, De Pinto V, Castellani L: Intracellular localization and isoform expression of the voltage-dependent anion channel (VDAC) in normal and dystrophic skeletal muscle. J Muscle Res Cell Motil 2000;21:433-442.

-33 Majumder S, Fisk HA: VDAC3 and Mps1 negatively regulate ciliogenesis. Cell Cycle 2013;12:849-858.

34 Saeed U, Durgadoss L, Valli RK, Joshi DC, Joshi PG, Ravindranath V: Knockdown of cytosolic glutaredoxin 1 leads to loss of mitochondrial membrane potential: implication in neurodegenerative diseases. PLoS One 2008;3:e2459.

35 Madesh M, Hajnoczky G: VDAC-dependent permeabilization of the outer mitochondrial membrane by superoxide induces rapid and massive cytochrome c release. J Cell Biol 2001;155:1003-1015.

-36 Han D, Antunes F, Canali R, Rettori D, Cadenas E: Voltage-dependent anion channels control the release of the superoxide anion from mitochondria to cytosol. J Biol Chem 2003;278: 5557-5563.

37 Sun Y, Vashisht AA, Tchieu J, Wohlschlegel JA, Dreier L: Voltage-dependent anion channels (VDACs) recruit Parkin to defective mitochondria to promote mitochondrial autophagy. J Biol Chem 2012;287:4065240660.

- 38 Geisler S, Holmström KM, Skujat D, Fiesel FC, Rothfuss OC, Kahle PJ, Springer W: PINK1/Parkin-mediated mitophagy is dependent on VDAC1 and p62/SQSTM1. Nat Cell Biol 2010;12:119-31.

-39 Sultana R, Boyd-Kimball D, Poon HF, Cai J, Pierce WM, Klein JB, Merchant M, Markesbery WR, Butterfield DA. Redox proteomics identification of oxidized proteins in Alzheimer's disease hippocampus and cerebellum: an approach to understand pathological and biochemical alterations in AD. Neurobiol Aging 2006;27:1564-1576. 